Pacific Journal of Mathematics

REAL ANALYTIC REGULARITY OF THE SZEGO

SO-CHN CHIN 


\section{REAL ANALYTIC REGULARITY \\ OF THE SZEGÖ PROJECTION ON CIRCULAR DOMAINS}

\section{So-Chin Chen}

In this note we show that $D \subseteq \mathbb{C}^{n}, n \geq 2$, is a smooth bounded pseudoconvex domain with real analytic defining function $r(z)$ such that $\sum_{k=1}^{n} z_{k}\left(\partial r / \partial z_{k}\right) \neq 0$ holds near some $x_{0} \in b D$, then if $g \in$ $C^{\omega}(b D)$, we have that the Szegö projection of $g, S g$, is real analytic near $x_{0}$. In particular if $D$ is a smooth bounded complete Reinhardt (or Reinhardt) pseudoconvex domain with real analytic boundary, then the Szegö projection $S$ preserves real analyticity globally.

I. Introduction. Let $D \subseteq \mathbb{C}^{n}, n \geq 2$, be a smooth bounded pseudoconvex domain. Denote by $L^{2}(b D)$ the space of square-integrable functions on the boundary and by $H^{2}(b D)$ the closed subspace of $L^{2}(b D)$ whose Poisson integrals are holomorphic in $D$. Then we define the Szegö projection $S$ to be the orthogonal projection from $L^{2}(b D)$ onto $H^{2}(b D)$. It is represented by integration against the Szegö kernel function $S(\omega, z)$, i.e., for $f \in L^{2}(b D)$, we have

$$
S f(\omega)=\int_{b D} S(\omega, z) f(z) d \sigma_{z}
$$

The smooth regularity of the Szegö projection for a large class of domains has been established, for instance, see [1] [2], [3], [4], [8], [9], [10], [12]. Hence in this paper we are going to study the real analytic regularity of the Szegö projection on circular domains. The problems can be formulated as follows.

1. (Global version): Suppose that the boundary of $D$ is real analytic, then does the Szegö projection $S$ map analytic functions to analytic functions, i.e., $S: C^{\omega}(b D) \rightarrow C^{\omega}(b D)$ ?

2. (Local version): Suppose that the boundary of $D$ is real analytic near some point $x_{0} \in b D$, then does the Szegö projection $S$ preserve real analyticity near $x_{0}$ ?

These problems are quite open. The only results we know so far are due to D. Tartakoff [13]. He showed the following. 
THEOREM (Tartakoff). On a real, real analytic CR-manifold of dimension $2 n-1$ whose Levi form is non-degenerate and which satisfies $Y(q)$, then $\square_{b}$ is locally real analytic hypoelliptic on $(p, q)$-forms.

For global result in this case see also Tartakoff [14].

The condition $Y(q)$ means that the Levi form has $\max (q+1, n-q)$ eigenvalues of the same sign or $\min (q+1, n-q)$ pairs of eigenvalues of opposite sign at each point. Therefore if one has a smooth bounded strictly pseudoconvex domain $D$ in $\mathbb{C}^{n}, n \geq 3$, with real analytic boundary, then one can apply the following formula

$$
S=\mathrm{Id}-\bar{\partial}_{b}^{*} N_{b} \bar{\partial}_{b},
$$

where $N_{b}$ is the boundary Neumann operator, to show that the Szegö projection $S$ preserves real analyticity locally (hence globally too). However, we must point out here that this theorem does not apply to the domains in $\mathbb{C}^{2}$, because condition $Y(1)$ is violated on such domains.

Very recently M. Derridj and D. Tartakoff [7] showed that if the defining function near $0 \in b D$ can be expressed as

$$
\operatorname{Im} \omega=h\left(|z|^{2}\right)
$$

with $h$ real analytic and $h(0)=0$, then again a local theorem holds near $0 \in b D$.

In this paper we prove the following main results.

Theorem 1. Let $D \subseteq \mathbb{C}^{n}, n \geq 2$, be a smooth bounded pseudoconvex circular domain with real analytic defining function $r(z)$. Suppose that $\sum_{k=1}^{n} z_{k}\left(\partial r / \partial z_{k}\right) \neq 0$ holds near some $x_{0} \in b D$ and that $f$ is globally real analytic, i.e., $f \in C^{\omega}(b D)$. Then $S f$ is real analytic near $x_{0}$.

We remark here that (i) Theorem 1 is also true in dimension two, (ii) it is not quite a local theorem, because we need $f$ to be globally real analytic. It follows from Theorem 1 that we have

Corollary 2. Let $D \subseteq \mathbb{C}^{n}, n \geq 2$, be a smooth bounded pseudoconvex circular domain with real analytic defining function $r(z)$. If $\sum_{k=1}^{n} z_{k}\left(\partial r / \partial z_{k}\right) \neq 0$ holds for all $z \in b D$, then the Szegö projection preserves real analyticity globally. 
Since the transversal condition always holds on a complete Reinhardt domain, see Chen [6], we have

Corollary 3. Let $D \subseteq \mathbb{C}^{n}, n \geq 2$, be a smooth bounded complete Reinhardt pseudoconvex domain with real analytic boundary. Then the Szegö projection preserves real analyticity globally.

Next if we drop the transversal condition, we can show

TheOREM 4. Let $D \subseteq \mathbb{C}^{n}, n \geq 2$, be a smooth bounded Reinhardt pseudoconvex domain with real analytic boundary. Then the Szegö projection preserves real analyticity globally.

The author would like to thank Mei-chi Shaw for bringing this problem to his attention.

II. Proof of the main results. A domain $D$ in $\mathbb{C}^{n}$ is called circular if $\left(z_{1}, \ldots, z_{n}\right) \in D$ implies $\left(e^{i \theta} z_{1}, \ldots, e^{i \theta} z_{n}\right) \in D$ for all $\theta \in \mathbb{R}$, and $D$ is called Reinhardt (or multi-circular) if $\left(z_{1}, \ldots, z_{n}\right) \in D$ implies $\left(e^{i \theta_{1}} z_{1}, \ldots, e^{i \theta_{n}} z_{n}\right) \in D$ for $\theta_{1}, \ldots, \theta_{n} \in \mathbb{R} . D$ is called complete Reinhardt if $\left(z_{1}, \ldots, z_{n}\right) \in D$ implies $\left(\omega_{1}, \ldots, \omega_{n}\right) \in D$ with $\left|\omega_{j}\right| \leq\left|z_{j}\right|$ for all $j=1, \ldots, n$.

First we recall that the Szegö kernel function $S(\omega, z)$ can be represented as follows. Let $\left\{\varphi_{j}\right\}_{j=1}^{\infty}$ be an orthonormal basis for $H^{2}(b D)$. Here we have identified each element $f \in H^{2}(b D)$ with its Poisson integral. Then we have

$$
S(\omega, z)=\sum_{j=1}^{\infty} \varphi_{j}(\omega) \overline{\varphi_{j}(z)}, \quad \forall \omega, z \in D,
$$

and the expression is independent of the choice of the basis.

Next we prove some basic facts on circular domains. Define an $S^{1}$-action on $D$ as follows,

$$
\begin{aligned}
\pi: S^{1} \times \bar{D} & \rightarrow \bar{D}, \\
\left(e^{i \theta}, z\right) & \mapsto \omega=e^{i \theta} \cdot z=\left(e^{i \theta} z_{1}, \ldots, e^{i \theta} z_{n}\right) .
\end{aligned}
$$

Then for each fixed $\theta, \pi_{b}=\left.\pi\right|_{b D}$ is a CR-diffeomorphism of $b D$.

LEMMA 2.2. Let $D \subseteq \mathbb{C}^{n}, n \geq 2$, be a smooth bounded circular domain. Then for each fixed $\theta$ we have $\pi_{b}^{*} d \sigma_{\omega}=d \sigma_{z}$, where $d \sigma$ denotes the surface element on $b D$. 
Proof. Let $r(\omega)$ be the defining function for $D_{\omega}$. Here we use subscripts to emphasize the domain we consider. Then $r \circ \pi(z)$ will be a defining function for $D_{z}$. By using the $*$-operator (cf. [11]), we have

$$
\pi_{b}^{*} d \sigma_{\omega}=\pi_{b}^{*}\left(\frac{2}{\|d r\|} i_{\omega}^{*}(* \partial r)\right)
$$

where $i: D \hookrightarrow \mathbb{C}^{n}$ is the inclusion map. Then by direct computation, we get

$$
\begin{aligned}
\pi_{b}^{*} d \sigma_{\omega} & =\frac{2}{\|d(r \circ \pi)\|} \pi_{b}^{*} i_{\omega}^{*}\left(\sum_{k=1}^{n} \frac{1}{2^{n-1} \cdot i^{n}} \frac{\partial r}{\partial \omega_{k}}(\omega) d \omega_{k} \bigwedge_{j \neq k}\left(d \bar{\omega}_{j} \wedge d \omega_{j}\right)\right) \\
& =\frac{2}{\|d(r \circ \pi)\|} i_{z}^{*} \cdot \pi^{*}\left(\sum_{k=1}^{n} \frac{1}{2^{n-1} \cdot i^{n}} \frac{\partial r}{\partial \omega_{k}}(\omega) d \omega_{k} \bigwedge_{j \neq k}\left(d \bar{\omega}_{j} \wedge d \omega_{j}\right)\right) \\
& =\frac{2}{\|d(r \circ \pi)\|} i_{z}^{*}(* \partial(r \circ \pi)) \\
& =d \sigma_{z} .
\end{aligned}
$$

This completes the proof of the lemma.

From Lemma 2.2 and the representation of the Szegö kernel function we obtain immediately the following transformation law for the Szegö kernel on circular domains.

LEMMA 2.3. Under the hypotheses of Lemma 2.2, we have $S(\omega, z)=$ $S(\pi(\omega), \pi(z))$.

Now we define the crucial vector field $T$ on $b D$ as follows. Let $z$ be a point near the boundary. Define

$$
\begin{aligned}
\Lambda_{z}: S^{1} & \rightarrow \bar{D} \\
e^{i \theta} & \mapsto e^{i \theta} \cdot z=\left(e^{i \theta} z_{1}, \ldots, e^{i \theta} z_{n}\right),
\end{aligned}
$$

and denote by $\Lambda_{z, *}$ the differential mapping of $\Lambda_{z}$. Then define the vector field $T$ at $z$ by

$$
\begin{aligned}
T(z) & =\frac{1}{2} \Lambda_{z, *}\left(\left.\frac{\partial}{\partial \theta}\right|_{\theta=0}\right)=\frac{i}{2} \sum_{j=1}^{n} z_{j} \frac{\partial}{\partial z_{j}}-\frac{i}{2} \sum_{j=1}^{n} \bar{z}_{j} \frac{\partial}{\partial \bar{z}_{j}} \\
& =\frac{i}{2} X-\frac{i}{2} \bar{X}
\end{aligned}
$$

where $X=\sum_{j=1}^{n} z_{j}\left(\partial / \partial z_{j}\right)$. 
The vector field $T$ defined in this way has many nice properties. First of all, if $\left(\partial r / \partial z_{n}\right)\left(x_{0}\right) \neq 0$, then

$$
L_{k}=\frac{\partial r}{\partial z_{k}} \frac{\partial}{\partial z_{n}}-\frac{\partial r}{\partial z_{n}} \frac{\partial}{\partial z_{k}}, \quad k=1, \ldots, n-1,
$$

forms a local basis for $T^{1,0}(b D)$. Hence by assumption $X r \neq 0$ near $x_{0}, L_{1}, \ldots, L_{n-1}, \bar{L}_{1}, \ldots, \bar{L}_{n-1}$ and $T$ form a basis for complex tangent space on $b D$ near $x_{0}$, and it is shown in [6] that we have (2.4) $\left[T, L_{k}\right]=-i L_{k}, \quad\left[T, \bar{L}_{k}\right]=i \bar{L}_{k}, \quad$ for $k=1, \ldots, n-1$.

The next lemma shows that $T$ commutes with the Szegö projection $S$ on such domains.

LEMMA 2.5. Under the hypotheses of Theorem 1, we have $T S u=$ $S T u$ for all $u \in C^{\infty}(b D)$.

Proof. Since $S u \in C^{\infty}(b D)$, one can apply $T$ to $S u$. Also by the construction of $T$, we get by writing $z=e^{i t} \cdot \eta$,

$$
\begin{aligned}
2 T S u(\omega) & =\left.\frac{d}{d t} \int_{b D} S\left(e^{i t} \cdot \omega, z\right) u(z) d \sigma_{z}\right|_{t=0} \\
& =\left.\frac{d}{d t} \int_{b D} S\left(e^{i t} \cdot \omega, e^{i t} \cdot \eta\right) u\left(e^{i t} \cdot \eta\right) d \sigma_{\eta}\right|_{t=0} \\
& =\left.\frac{d}{d t} \int_{b D} S(\omega, \eta) u\left(e^{i t} \cdot \eta\right) d \sigma_{\eta}\right|_{t=0} \\
& =2 S T u(\omega) .
\end{aligned}
$$

This completes the proof of the lemma.

Now we begin to prove Theorem 1. So we assume that $f \in C^{\omega}(b D)$. It follows from the smooth regularity of the Szegö projection that we have $S f \in C^{\infty}(b D)$. Denote by $L$ (or $\bar{L}$ ) any of the $L_{i}$ 's (or $\bar{L}_{i}$ 's) with $i \leq n-1$, and by $\stackrel{(-)}{L}$ any of $L_{i}$ 's or $\bar{L}_{i}$ 's with $i \leq n-1$. Also denote by $Z$ either $\stackrel{(-)}{L}$ or $T$. Denote by $a_{(1)}$ any of the finite collection of analytic functions that occur in commutators of $L, \bar{L}$ and $T$ and integration by parts. Let $a_{(j+1)}=a_{(1)} \cdot a_{(j)}$ or $a_{(j+1)}=$ $Z a_{(j)}$. Hence there exists a constant $R>0$ such that for all $k \in \mathbb{N}$, we have

$$
\left|a_{(k)}\right| \leq R R^{k} k !
$$


The proof for Theorem 1 will be complete if one can show

$$
\left\|Z_{1} \cdots Z_{q} S f\right\|_{L^{2}(U)} \leq M M^{q} q !,
$$

for all $q \in \mathbb{N}$ and some open neighborhood $U$ of $x_{0}$ and some $M>$ 0 uniformly in $q$. We may assume that $X r \neq 0$ holds on some neighborhood of $\bar{U}$.

Let $\varphi$ be a cut-off function with $\varphi \equiv 1$ in some neighborhood of $x_{0}$ and $\operatorname{supp} \varphi$ contained in $U$. Denote by $\varphi^{\prime}$ any first derivative of $\varphi$. Then by Lemma 2.5 one can estimate the pure terms quite easily,

$$
\left\|\varphi T^{p} S f\right\|=\left\|\varphi S T^{p} f\right\| \leq\left\|T^{p} f\right\| \leq R_{f} R_{f}^{p} p !,
$$

for some $R_{f}>0$. To estimate the mixed terms we use $\mathrm{Op}(k, q)$ to denote any differential operator of order $q$ formed out of the $\stackrel{(-)}{L}$ and $T$ in all order with precisely $k \stackrel{(-)}{L}$ 's. Thus if $k=0$, by (2.8), we have

$$
\|\varphi \mathrm{Op}(0, q) S f\|=\left\|\varphi T^{q} S f\right\| \leq R_{f} R_{f}^{q} q ! .
$$

Hence what remains in this note is to estimate the term $\mathrm{Op}(s, q) S f$ with $s \geq 1$. We will use underline to mean at most such terms are being considered and $c$ is a constant depending only on $n$. First we write

$$
\begin{aligned}
\mathrm{Op}(s, p)= & T^{r}{ }^{(-)} \mathrm{Op}(s-1, p-r-1) \\
= & \stackrel{(-)}{L} T^{r} \mathrm{Op}(s-1, p-r-1) \\
& +\sum_{j=1}^{r}( \pm) i T^{r-j}{ }^{(-)} L T^{j-1} \mathrm{Op}(s-1, p-r-1) \\
= & \stackrel{(-)}{L} \mathrm{Op}(s-1, p-1)+\sum_{j=1}^{r}( \pm) i \mathrm{Op}(s, p-1) .
\end{aligned}
$$

Define

$$
\begin{aligned}
I_{p}(\varphi)= & \|\varphi \mathrm{Op}(s, p) S f\|+\|\varphi T \mathrm{Op}(s-1, p-1) S f\| \\
& +\sum_{i=1}^{n-1}\left(\left\|\varphi L_{i} \mathrm{Op}(s-1, p-1) S f\right\|\right. \\
& \left.\quad+\left\|\varphi \bar{L}_{i} \mathrm{Op}(s-1, p-1) S f\right\|\right) .
\end{aligned}
$$

By (2.9) we see that

$$
\begin{aligned}
I_{p}(\varphi) \leq & 2 \sum_{i=1}^{n-1}\left(\left\|\varphi L_{i} \mathrm{Op}(s-1, p-1) S f\right\|+\left\|\varphi \bar{L}_{i} \mathrm{Op}(s-1, p-1) S f\right\|\right) \\
& +\|\varphi T \mathrm{Op}(s-1, p-1) S f\|+(p-1)\|\varphi \mathrm{Op}(s, p-1) S f\|
\end{aligned}
$$


We estimate the first term as follows:

$$
\begin{aligned}
\| \varphi L O \mathrm{Op} & (s-1, p-1) S f \|^{2} \\
= & (\varphi L \mathrm{Op}(s-1, p-1) S f, \varphi L \mathrm{Op}(s-1, p-1) S f) \\
=\| & \varphi \bar{L} \mathrm{Op}(s-1, p-1) S f \|^{2} \\
& +(\varphi \mathrm{Op}(s-1, p-1) S f,-\varphi[\bar{L}, L] \mathrm{Op}(s-1, p-1) S f) \\
& +2\left(\varphi^{\prime} \mathrm{Op}(s-1, p-1) S f, \varphi L \mathrm{Op}(s-1, p-1) S f\right) \\
& +2\left(\varphi^{\prime} \mathrm{Op}(s-1, p-1) S f, \varphi \bar{L} \mathrm{Op}(s-1, p-1) S f\right) \\
& +\left(a_{(1)} \varphi \mathrm{Op}(s-1, p-1) S f, \varphi L \mathrm{Op}(s-1, p-1) S f\right) \\
& +\left(a_{(1)} \varphi \mathrm{Op}(s-1, p-1) S f, \varphi \bar{L} \mathrm{Op}(s-1, p-1) S f\right) .
\end{aligned}
$$

It shows that for $C_{1}>0$ we have

$$
\begin{aligned}
\|\varphi L O p(s-1, p-1) S f\| & \\
\leq & \|\varphi \bar{L} \mathrm{Op}(s-1, p-1) S f\| \\
& +C_{1}(\|\varphi \mathrm{Op}(s-1, p-1) S f\| \\
& +4\left\|\varphi^{\prime} \mathrm{Op}(s-1, p-1) S f\right\| \\
& \left.+2\left\|a_{(1)} \varphi \mathrm{Op}(s-1, p-1) S f\right\|\right) \\
& +\frac{1}{C_{1}}(3\|\varphi L \mathrm{Op}(s-1, p-1) S f\|+3\|\varphi \bar{L} \mathrm{Op}(s-1, p-1) S f\| \\
& +\|\varphi[\bar{L}, L] \mathrm{Op}(s-1, p-1) S f\|) .
\end{aligned}
$$

LEMMA 2.11. (i) $[\bar{L}, L]=\underline{(2 n-1)} a_{(1)} Z$.

(ii)

$$
\begin{aligned}
\bar{L} \mathrm{Op}(s-1, p-1)= & \operatorname{Op}(s-1, p-1) \bar{L} \\
& +\sum_{j=1}^{p-1}(2(2 n-1))^{j}\left(\begin{array}{c}
p-1 \\
j
\end{array}\right) a_{(j)} Z_{j} Z_{j+1} \cdots Z_{p-1},
\end{aligned}
$$

where $Z_{j}$ denotes any $Z$. Hence one may choose $c=2(2 n-1)$.

Proof. (i) is trivial. For (ii) we have

$$
\begin{aligned}
& {[\bar{L}, \mathrm{Op}(s-1, p-1)]=\left[\bar{L}, Z_{1} \cdots Z_{p-1}\right]} \\
& \quad=\sum_{j=1}^{p-1}\left(\begin{array}{c}
p-1 \\
j
\end{array}\right)\left[\left[\cdots\left[\bar{L}, Z_{1}\right] \cdots\right], Z_{j}\right] Z_{j+1} \cdots Z_{p-1} .
\end{aligned}
$$

Hence the conclusion follows immediately. 
Since $S f$ is annihilated by $\bar{L}$, we obtain

$$
\begin{aligned}
I_{p}(\varphi) \leq 2 \sum_{i=1}^{n-1}\left\{2\left\|\varphi \bar{L}_{i} \mathrm{Op}(s-1, p-1) S f\right\|\right. \\
+C_{1}(\|\varphi \mathrm{Op}(s-1, p-1) S f\| \\
+4\left\|\varphi^{\prime} \mathrm{Op}(s-1, p-1) S f\right\| \\
\left.\quad+2 R^{2}\|\varphi \mathrm{Op}(s-1, p-1) S f\|\right) \\
+\frac{1}{C_{1}}\left(3\left\|\varphi L_{i} \mathrm{Op}(s-1, p-1) S f\right\|\right. \\
+3\left\|\varphi \bar{L}_{i} \mathrm{Op}(s-1, p-1) S f\right\| \\
\left.\left.\quad+c R^{2}\|\varphi Z \mathrm{Op}(s-1, p-1) S f\|\right)\right\} \\
+\|\varphi T \mathrm{Op}(s-1, p-1) S f\| \\
+(p-1)\|\varphi \mathrm{Op}(s, p-1) S f\| .
\end{aligned}
$$

Choose $C_{1}=\max \left(24,8 n c R^{2}\right)$; then we get

$$
\begin{aligned}
I_{p}(\varphi) \leq & 8 \sum_{i=1}^{n-1} \sum_{j=1}^{p-1} c^{j}\left(\begin{array}{c}
p-1 \\
j
\end{array}\right)\left\|a_{(j)} \varphi Z_{j} Z_{j+1} \cdots Z_{p-1} S f\right\| \\
& +4(n-1) C_{1}(\|\varphi \mathrm{Op}(s-1, p-1) S f\| \\
& \quad+4\left\|\varphi^{\prime} \mathrm{Op}(s-1, p-1) S f\right\| \\
& \left.\quad+2 R^{2}\|\varphi \mathrm{Op}(s-1, p-1) S f\|\right) \\
& +2\|\varphi T \mathrm{Op}(s-1, p-1) S f\| \\
& +2(p-1)\|\varphi \mathrm{Op}(s, p-1) S f\| \\
\leq & 8(n-1) \sum_{j=1}^{p-1} c^{j}\left(\begin{array}{c}
p-1 \\
j
\end{array}\right) \cdot R R^{j} j ! I_{p-1}(\varphi) \\
& +16(n-1) C_{1} I_{p-1}\left(\varphi^{\prime}\right) \\
& +4(n-1) C_{1}\left(1+2 R^{2}\right) I_{p-1}(\varphi) \\
& +2\|\varphi \mathrm{Op}(s-1, p) S f\|+2(p-1) I_{p-1}(\varphi)
\end{aligned}
$$

Since the vector field $\stackrel{(-)}{L}$ in general is defined only locally, we need a special cut-off function which is of compact support, but behaves like an analytic function up to certain order.

LEMMA (Ehrenpreis). Let $x_{0} \in b D$ and $U_{1}, U_{2}$ be two neighborhoods of $x_{0}$ with $U_{1} \Subset U_{2} \Subset U$; then there exists a constant $M>0$ such that for any integer $k$ one can find $\varphi_{k} \in C_{0}^{\infty}\left(U_{2}\right)$ with 
$0 \leq \varphi_{k} \leq 1, \varphi_{k} \equiv 1$ on $U_{1}$ and satisfying

$$
\left|D^{\alpha} \varphi_{k}\right| \leq M(M k)^{|\alpha|}, \quad \text { for }|\alpha| \leq k+1 .
$$

Now one can replace $\varphi$ by $\varphi_{p}^{(b)}$, some derivative of $\varphi_{p}$ of order $\leq b$, and $p$ by $p-b$ in (2.13). Then we obtain

$$
\text { (2.15) } \begin{aligned}
I_{p-b}\left(\varphi_{p}^{(b)}\right) \leq & 8(n-1) \sum_{j=1}^{p-b-1} \frac{c^{j}\left(\begin{array}{c}
p-b-1 \\
j
\end{array}\right)}{}\left(R R^{j} j ! I_{p-b-j}\left(\varphi_{p}^{(b)}\right)\right. \\
& +16(n-1) C_{1} I_{p-b-1}\left(\varphi_{p}^{(b+1)}\right) \\
& +4(n-1) C_{1}\left(1+2 R^{2}\right) I_{p-b-1}\left(\varphi_{p}^{(b)}\right) \\
& +2\left\|\varphi_{p}^{(b)} \mathrm{Op}(s-1, p-b) S f\right\| \\
& +2(p-b-1) I_{p-b-1}\left(\varphi_{p}^{(b)}\right) .
\end{aligned}
$$

There are five terms in (2.15). Inductively we will show that for $p \geq b \geq 0$, we have

$$
I_{p-b}\left(\varphi_{p}^{(b)}\right) \leq R_{1}\left(R_{2} p\right)^{b}\left(R_{3} p\right)^{p-b} R_{4}^{s},
$$

where $R_{1}, R_{2}, R_{3}$ and $R_{4}$ are some constants, i.e., we will show that each term can be bounded by $\frac{1}{5} R_{1}\left(R_{2} p\right)^{b}\left(R_{3} p\right)^{p-b} R_{4}^{s}$. The initial step $s=0$ or $p=b$ is easy to check. Hence we assume that (2.16) is true for $b>b_{0}$ or $s<s_{0}$. Then we prove the case $b=b_{0}$ and $s=s_{0}$.

$$
\begin{aligned}
\text { Term } 1 \leq & 8(n-1) \sum_{j=1}^{p-b_{0}-1} \frac{\left(p-b_{0}-1\right) !}{\left(p-b_{0}-j-1\right) ! j !} \\
= & R_{1}\left(R_{2} p\right)^{b_{0}}\left(R_{3} p\right)^{p-b_{0}} \cdot R_{4}^{s_{0}} \\
& \cdot \sum_{j=1}^{p-b_{0}-1} \frac{\left(p-b_{0}-1\right) !}{\left(p-b_{0}-j-1\right) ! p^{j}} \cdot \frac{8(n-1) R(c R)^{j}}{R_{3}^{j}} \\
\leq & \frac{1}{5} R_{1}\left(R_{2} p\right)^{b_{0}}\left(R_{3} p\right)^{p-b_{0}} \cdot R_{4}^{s_{0}},
\end{aligned}
$$

provided $R_{3}$ is chosen large enough.

$$
\begin{aligned}
\text { Term } 2 & \leq 16(n-1) C_{1} R_{1}\left(R_{2} p\right)^{b_{0}+1} \cdot\left(R_{3} p\right)^{p-b_{0}-1} \cdot R_{4}^{s_{0}} \\
& =R_{1}\left(R_{2} p\right)^{b_{0}} \cdot\left(R_{3} p\right)^{p-b_{0}} \cdot R_{4}^{S_{0}} \cdot\left(\frac{16(n-1) C_{1} R_{2}}{R_{3}}\right) \\
& \leq \frac{1}{5} R_{1}\left(R_{2} p\right)^{b_{0}}\left(R_{3} p\right)^{p-b_{0}} \cdot R_{4}^{s_{0}},
\end{aligned}
$$

provided $R_{3} \geq 80(n-1) C_{1} R_{2}$. 


$$
\text { Term } \begin{aligned}
3 & \leq 4(n-1) C_{1}\left(1+2 R^{2}\right) \cdot R_{1}\left(R_{2} p\right)^{b_{0}}\left(R_{3} p\right)^{p-b_{0}-1} \cdot R_{4}^{s_{0}} \\
& =R_{1}\left(R_{2} p\right)^{b_{0}}\left(R_{3} p\right)^{p-b_{0}} \cdot R_{4}^{s_{0}} \cdot\left(\frac{4(n-1) C_{1}\left(1+2 R^{2}\right)}{R_{3} p}\right) \\
& \leq \frac{1}{5} R_{1}\left(R_{2} p\right)^{b_{0}}\left(R_{3} p\right)^{p-b_{0}} \cdot R_{4}^{s_{0}},
\end{aligned}
$$

provided $R_{3} \geq 20(n-1) C_{1}\left(1+2 R^{2}\right)$.

$$
\begin{aligned}
\text { Term } 4 & \leq 2 R_{1}\left(R_{2} p\right)^{b_{0}}\left(R_{3} p\right)^{p-b_{0}} \cdot R_{4}^{s_{0}-1} \\
& \leq \frac{1}{5} R_{1}\left(R_{2} p\right)^{b_{0}}\left(R_{3} p\right)^{p-b_{0}} \cdot R_{4}^{s_{0}}
\end{aligned}
$$

provided $R_{4} \geq 10$.

$$
\text { Term } \begin{aligned}
5 & \leq 2\left(p-b_{0}-1\right) R_{1}\left(R_{2} p\right)^{b_{0}}\left(R_{3} p\right)^{p-b_{0}-1} \cdot R_{4}^{s_{0}} \\
& =R_{1}\left(R_{2} p\right)^{b_{0}}\left(R_{3} p\right)^{p-b_{0}} \cdot R_{4}^{s_{0}}\left(\frac{2\left(p-b_{0}-1\right)}{R_{3} p}\right) \\
& \leq \frac{1}{5} R_{1}\left(R_{2} p\right)^{b_{0}}\left(R_{3} p\right)^{p-b_{0}} \cdot R_{4}^{s_{0}},
\end{aligned}
$$

provided $R_{3} \geq 10$. This completes the proof of (2.16). In particular, we have shown that for $k \leq p$,

$$
\begin{aligned}
\left\|\varphi_{p} \mathrm{Op}(k, p) S f\right\| & \leq I_{p}\left(\varphi_{p}\right) \leq R_{1}\left(R_{3} p\right)^{p} R_{4}^{k} \\
& \leq R_{1}\left(R_{3} R_{4} p\right)^{p} \\
& \leq M M^{p} p !
\end{aligned}
$$

for some large constant $M>0$ depending only on $R_{1}, R_{3}$ and $R_{4}$. This also completes the proof of Theorem 1.

To prove Theorem 4 one can apply the above techniques almost verbally with slight modification. The key point is that for every point $x_{0} \in b D$ we have to choose a vector field like $T$ for Theorem 1 which is transversal to $T^{1,0}(b D) \oplus T^{0,1}(b D)$ locally near $x_{0}$ and commutes nicely with $\stackrel{(-)}{L}$ and the Szegö projection. This can be done easily. By rotational symmetry of the domain one can choose a direction, say $n$, such that $\left(z_{n}\left(\partial r / \partial z_{n}\right)\right)\left(x_{0}\right) \neq 0$ holds in some neighborhood of $x_{0}$. Then define $S^{1}$-action on $\bar{D}$ as follows.

$$
\begin{aligned}
\pi: S^{1} \times \bar{D} & \rightarrow \bar{D} \\
\left(e^{i \theta}, z\right) & \mapsto e^{i \theta} \cdot z=\left(z_{1}, \ldots, z_{n-1}, e^{i \theta} z_{n}\right) .
\end{aligned}
$$

Such an action will generate a vector field $T_{n}=i z_{n}\left(\partial / \partial z_{n}\right)-$ $i \bar{z}_{n}\left(\partial / \partial \bar{z}_{n}\right)$ with the desired properties. So we are done. For more details in this case see [5]. 


\section{REFERENCES}

[1] H. P. Boas, Regularity of the Szegö projection in weakly pseudoconvex domains, Indiana Univ. Math. J., 34 (1985), 217-223.

[2] - The Szegö projection: Sobolev estimates in regular domains, Trans. Amer. Math. Soc., 300 (1987), 109-132.

[3] H. P. Boas, S. C. Chen and E. J. Straube, Exact regularity of the Bergman and Szegö projections on domains with partially transverse symmetries, Manuscripta Math., 62 (1988), 467-475.

[4] L. Boutet de Monvel and J. Sjöstrand, Sur la singularité des noyaux de Bergman et de Szegö, Astérisque, 34-35 (1976), 123-164.

[5] S. C. Chen, Global real analyticity of solutions to the $\bar{\partial}-$ Neumann problem on Reinhardt domains, Indiana Univ. Math. J., 37, No. 2 (1988), 421-430.

[6] __ Global analytic hypoellipticity of the $\bar{\partial}$-Neumann problem on circular domains, Invent. Math., 92 (1988), 173-185.

[7] M. Derridj and D. S. Tartakoff, Local analyticity for $\square_{b}$ and the $\bar{\partial}$-Neumann problem at certain weakly pseudoconvex points, Comm. Partial Differential Equations, 13 (12) (1988), 1521-1600.

[8] N. Kerzman and E. M. Stein, The Szegö kernel in terms of Cauchy-Fantappiè kernels, Duke Math. J., 45 (1978), 197-224.

[9] J. J. Kohn, Boundaries of Complex Manifolds, in Proc. Conference on Complex Analysis (Minneapolis), Springer-Verlag, New York, 1965.

[10] D. H. Phong and E. M. Stein, Estimates for the Bergman and Szegö projections on strongly pseudo-convex domains, Duke Math. J., 44 (1977), 695-704.

[11] R. M. Range, Holomorphic Functions and Integral Representations in Several Complex Variables, Springer-Verlag, New York, 1986.

[12] E. J. Straube, Exact regularity of Bergman, Szegö and Sobolev space projections in non-pseudoconvex domains, Math. Zeit., 192 (1986), 117-128.

[13] D. S. Tartakoff, On the local real analyticity of solutions to $\square_{b}$ and the $\bar{\partial}$ Neumann problem, Acta Math., 145 (1980), 177-204.

[14] _ On the global real analyticity of solutions to $\square_{b}$, Comm. Partial Differential Equations, 1 (1976), 283-311.

Received April 10, 1989. Partially supported by the NSF.

SUNY

AlbaNY, NY 12222 



\section{PACIFIC JOURNAL OF MATHEMATICS EDITORS}

\author{
V. S. VARADARAJAN \\ (Managing Editor) \\ University of California \\ Los Angeles, CA 90024-1555-05 \\ Herbert Clemens \\ University of Utah \\ Salt Lake City, UT 84112 \\ THOMAS ENRIGHT \\ University of California, San Diego \\ La Jolla, CA 92093
}

R. FINN

Stanford University

Stanford, CA 94305

HeRmann FlaschKa

University of Arizona

Tucson, AZ 85721

VAUghan F. R. Jones

University of California

Berkeley, CA 94720

SteVEN KeRCKHOFF

Stanford University

Stanford, CA 94305

\section{C. MOORE}

University of California

Berkeley, CA 94720

MARTIN SCHARLEMANN

University of California

Santa Barbara, CA 93106

HAROLD STARK

University of California, San Diego

La Jolla, CA 92093

\section{ASSOCIATE EDITORS \\ R. ARENS \\ E. F. BECKENBACH \\ B. H. NeUmanN \\ F. WoLF \\ (1904-1989) \\ K. Yoshida (1906-1982)}

\section{SUPPORTING INSTITUTIONS}

UNIVERSITY OF ARIZONA

UNIVERSITY OF BRITISH COLUMBIA

CALIFORNIA INSTITUTE OF TECHNOLOGY

UNIVERSITY OF CALIFORNIA

MONTANA STATE UNIVERSITY

UNIVERSITY OF NEVADA, RENO

NEW MEXICO STATE UNIVERSITY

OREGON STATE UNIVERSITY
UNIVERSITY OF OREGON

UNIVERSITY OF SOUTHERN CALIFORNIA

STANFORD UNIVERSITY

UNIVERSITY OF HAWAII

UNIVERSITY OF TOKYO

UNIVERSITY OF UTAH

WASHINGTON STATE UNIVERSITY

UNIVERSITY OF WASHINGTON

The Supporting Institutions listed above contribute to the cost of publication of this Journal, but they are not owners or publishers and have no responsibility for its content or policies.

Mathematical papers intended for publication in the Pacific Journal of Mathematics should be in typed form or offset-reproduced (not dittoed), double spaced with large margins. Please do not use built up fractions in the text of the manuscript. However, you may use them in the displayed equations. Underline Greek letters in red, German in green, and script in blue. The first paragraph must be capable of being used separately as a synopsis of the entire paper. In particular it should contain no bibliographic references. Please propose a heading for the odd numbered pages of less than 35 characters. Manuscripts, in triplicate, may be sent to any one of the editors. Please classify according to the 1991 Mathematics Subject Classification scheme which can be found in the December index volumes of Mathematical Reviews. Supply name and address of author to whom proofs should be sent. All other communications should be addressed to the managing editor, or Elaine Barth, University of California, Los Angeles, California 90024-1555-05.

There are page-charges associated with articles appearing in the Pacific Journal of Mathematics. These charges are expected to be paid by the author's University, Government Agency or Company. If the author or authors do not have access to such Institutional support these charges are waived. Single authors will receive 50 free reprints; joint authors will receive a total of 100 free reprints. Additional copies may be obtained at cost in multiples of 50 .

The Pacific Journal of Mathematics (ISSN 0030-8730) is published monthly except for July and August. Regular subscription rate: $\$ 190.00$ a year (10 issues). Special rate: $\$ 95.00$ a year to individual members of supporting institutions.

Subscriptions, orders for numbers issued in the last three calendar years, and changes of address should be sent to Pacific Journal of Mathematics, P.O. Box 969, Carmel Valley, CA 93924, U.S.A. Old back numbers obtainable from Kraus Periodicals Co., Route 100, Millwood, NY 10546.

The Pacific Journal of Mathematics at P.O. Box 969, Carmel Valley, CA 93924 (ISSN 0030-8730) is published monthly except for July and August. Second-class postage paid at Carmel Valley, California 93924, and additional mailing offices. Postmaster: send address changes to Pacific Journal of Mathématics, P.O. Box 969, Carmel Valley, CA 93924.

PUBLISHED BY PACIFIC JOURNAL OF MATHEMATICS, A NON-PROFIT CORPORATION Copyright (C) 1991 by Pacific Journal of Mathematics 


\section{Pacific Journal of Mathematics}

Vol. 148, No. $2 \quad$ April, 1991

Yuri A. Abramovich, Operators preserving disjointness on rearrangement

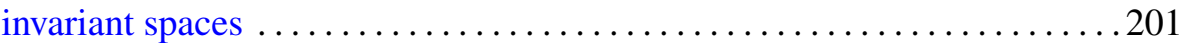

Andrew French Acker and Kirk Lancaster, Existence and geometry of a free boundary problem for the heat equation .................. 207

So-Chin Chen, Real analytic regularity of the Szegő projection on circular

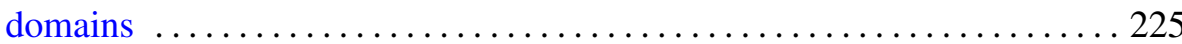

Chen-Lian Chuang, An independence property of central polynomials . . . 237

Peter Larkin Duren and M. Schiffer, Robin functions and energy

functionals of multiply connected domains $\ldots \ldots \ldots \ldots \ldots \ldots \ldots \ldots . \ldots 251$

Johan Henricus Bernardus Kemperman, Sets of uniqueness and systems

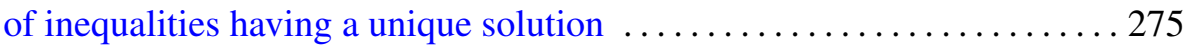

Ka-Lam Kueh, Fourier coefficients of nonholomorphic modular forms and sums of Kloosterman sums . .......................... 303

Gerard J. Murphy, Ordered groups and crossed products of $C^{*}$-algebras . . 319

You-Qiang Wang, The $p$-parts of Brauer character degrees in $p$-solvable

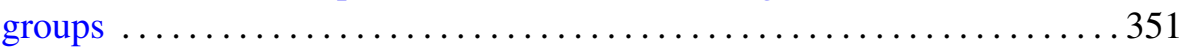

Hidenobu Yoshida, Harmonic majorization of a subharmonic function on a cone or on a cylinder ........................................ 369 